\title{
Whole-Genome Analysis of KPC-Producing Klebsiella Pneumoniae Isolates from Hospital Acquired Post-Neurosurgical Meningitis
}

\section{Mingyue Sun, Weiqiang Xiao and Qingxia $\mathrm{Xu}^{*}$}

Department of Clinical Laboratory, Affiliated Cancer Hospital of Zhengzhou University and Henan Cancer Hospital, Zhengzhou, Henan, People's Republic of

China

*Corresponding Author: Qingxia Xu, Department of Clinical Laboratory, Affiliated Cancer Hospital of Zhengzhou University and Henan Cancer Hospital, Zhengzhou, Henan, People's Republic of China.
Received: December 14, 2021

Published: January 31, 2022

(C) All rights are reserved by Qingxia Xu., et al.

\begin{abstract}
Background: Nosocomial bacterial infections from carbapenem-resistant Klebsiella pneumoniae (CRKP) are associated with high mortality in neurosurgical patients. This study examined the post-neurosurgical meningitis outbreak caused by CRKP of patients with nervous system tumours, and analysed the molecular characteristics of the causative strain.

Methods: Neurosurgical cancer patients with meningitis caused by CRKP between 2017-2019 were retrospectively analysed. Identification of strains and antimicrobial susceptibilities was conducted using BD Phoenix-100, 16S rRNA gene sequencing and broth microdilution. Multilocus sequence typing (MLST) and pulsed-field gel electrophoresis (PFGE) were used to identify the subtypes of $K$. pneumoniae. The genotype correlation, resistance genes and plasmid of isolates were assessed by whole-genome sequencing (WGS).

Results: Isolates were resistant to almost all of the tested antimicrobial agents except polymyxin and tigecycline. The PFGE and MLST revealed all isolates were the same strain - ST11-while WGS phylogenetic analysis indicated they were closely related. The isolates harboured blaKPC-2 and an IncFII-type plasmid; the blaKPC-2 gene had a similar genetic environment across isolates.

Conclusions: The results of molecular analysis showed that ST11 and IncFII-type plasmid in CRKP have close correlations and indicate a long-term retrospective genomic study throughout the hospital for KPC-producing K. pneumoniae is necessary.
\end{abstract}

Keywords: K. pneumoniae; Meningitis; Whole-Genome Sequencing; Incfii-Type Plasmid

\section{Introduction}

Patients (especially in nervous system cancer patients who receive cancer care) due to exposure to various risk factors (such as catheters and tubes, neurological deterioration, multiple injuries and damage to the immune system) and more susceptible to hospital infections [1-3]. During neurosurgical procedures, especially in the ICU, invading microorganisms overcome complex host defence mechanisms and cause infections with high morbidity and mortality [4-8]. Meningitis is several infections, defined as inflammation of the meninges; it may be caused by a variety of infectious agents, which include bacteria, viruses, parasites and fungi, as well as noncommunicable process. Although the most common viral meningitis, bacterial meningitis but may be more severe, potentially lifethreatening [9].

Last several years, carbapenem-resistant Klebsiella pneumoniae (CRKP) has become one of the biggest global public health concerns $[10,11]$. Despite this, there are few reports in the literature on meningitis caused by CRKP [12,13]. This study reports ten cases 
of post-neurosurgical nosocomial meningitis due to CRKP over three years; six isolate strains were further studied. From 2017 to 2019 , every July to September, there were nosocomial meningitis outbreaks in cancer patients of central nervous system in our hospital's neurosurgery department; the culprit was identified as CRKP. 30 pathogens were isolated from different sites on the patients, and six isolate strains were further studied and whole genome sequenced to identify the relationship between the strains. To our knowledge, this report is the first CRKP meningitis outbreak in cancer patients described in central China $[12,13]$.

\section{Materials and Methods}

Bacterial isolation, species identification and antimicrobial susceptibility profiles

Identification of strains was performed by using BD Phoenix-100 (BD, American) and 16S rRNA gene sequencing. Antimicrobial susceptibilities were initially tested using BD Phoenix-100 (BD, American), following which MICs were measured using broth microdilutions according to the Clinical and Laboratory Standards Institute (CLSI) guidelines [14]. Escherichia coli (E. coli) strain ATCC 25922 was used as the quality control. The CLSI breakpoints (M100-S27) and the 2018 EUCAST breakpoints (only for polymyxin and tigecycline) were used to interpret the MIC results.

Pulsed-field gel electrophoresis (PFGE) and Multilocus sequence typing (MLST)

All isolates for PFGE. Briefly, genomic DNA was digested with XbaI (Bio-Rad Laboratories, Hercules, CA, USA), restriction fragments were separated in CHEF Mapper XA System (Bio-Rad Laboratories). Cluster analysis using Bionumerics software (Applied Maths NV, Sint-Martens-Latem, East Flanders, Belgium) was performed with the Dice similarity coefficients and unweighted-pair group matching algorithm. The same PFGE pattern were defined in strains which shared with $>75 \%$ similarity.

The sequence types of the six carbapenemase producers were determined by multilocus sequence typing (MLST) analysis with seven housekeeping genes (gapA, infB, $m d h$, pgi, phoE, rpoB and $\operatorname{ton} B$ ) amplified and sequenced according to Diancourt., et al. Alleles and sequence types were assigned using the MLST database (http://www.pasteur.fr/mlst/Kpneumoniae.html) [15].

\section{Conjugation and plasmid analysis}

In plasmid conjugation experiments, E. coli J53 as a recipient strain were performed. Next, the transconjugants were cultured on agar (OXOID, UK) medium supplemented with $200 \mathrm{mg} / \mathrm{L}$ sodium azide and $2 \mathrm{mg} / \mathrm{L}$ meropenem. Finally, BD?Bruker MALDI Biotypersystem was used to identify transconjugants and amplify blaKPC by PCR to ensure that the plasmid was successfully transferred to the recipient strain. Plasmid comparisons were performed by using BRIG (http://brig.sourceforge.net/) and Easyfifig (http://mjsull.github.io/Easyfifig/) tools.

\section{WGS}

The preferred embodiment, genomic extracted with Wizard Genomic DNA Purification Kit (Promega, Madison, WI). Paired-end sequencing $(2 \times 150 \mathrm{bp})$ was used to obtain the whole genome sequence (WGS) of six strains in the Illumina Hiseq 2500 and Nanopore MinION (long-read) sequencing with Rapid Barcoding Kit (RBK004) in the R9.4.1 flow cell. As previously reported, use the hybrid de novo assembly strategy with Unicycler tools to assemble the genome [16]. Use different databases, including Plasmid Finder, Res Finder and ISfinder, to study the distribution of plasmid replicons, resistance genes and inserted sequences in the genome. The genetic environments surrounding the carbapenemase genes were annotated using Easyfifig 2.2.3.

\section{Phylogenetic Reconstruction and analysis}

Use kSNP program to identify core genome single nucleotide polymorphism SNPs on WGS data of six isolates [17]. kSNP is a programme in view of k-mer analysis. Before running kSNP, use Kchooser to evaluate the best value of k-mer. After running the kSNP program, use the output file for further analysis [18]. Use iTOL to generate the maximum likelihood tree output from the core SNP matrix of kSNP (https://itol.embl.de/).

\section{Nucleotide sequence accession numbers}

The sequences of the plasmid of blaKPC-2 were submitted to NCBI GenBank with accession numbers: pKP6106_KPC

(MW465706) and pKP7849_KPC (MW478298). All relevant data is available from the corresponding author upon reasonable request.

\section{Results}

\section{Clinical characteristics of CRKP meningitis}

The medical records of patients from whom CRKP were isolated are listed in Supplement table 1. A total of 10 cases bacterial meningitis and 30 pathogens were identified between July-August of 2017-2019. Although there were few confirmed cases, there are suspected outbreaks in neurosurgery department of our hospital 
from July to August every year. Multidrug-resistant bacteria with the same drug resistance spectrum were isolated from different sites from each patient, and the antimicrobial susceptibility patterns were tested (Supplement table 2). The strain isolated from patients 1-4 was resistant to all of the tested antimicrobial agents used for treatment of infections caused by Enterobacteriaceae, except chloramphenicol, minocycline, polymyxin and tigecycline. The strains isolated from patients 5-6 and patients 8-9 were susceptible to trimethoprim-sulfamethoxazole, minocycline, polymyxin and tigecycline, and to chloramphenicol, polymyxin and tigecycline, respectively. The strains isolated from patient 7 and patient 10 were susceptible to minocycline, polymyxin and tigecycline, and amikacin, polymyxin, and tigecycline, respectively.

Among the 26 K. pneumoniae causing meningitis, 20 strains of bacteria were not preserved and 6 isolates were available for further experiments, which were isolated from 6 different patients. The six strains include three from 2017, one from 2018 and two from 2019.

PFGE and multilocus sequence typing (MLST)

MLST analysis found that the six clinically derived carbapenemaseproducing K. pneumoniae were all ST11; this is the dominant clone of KPC-Kp and has been widely reported in different regions in China. The PFGE results confirmed the homology of the six isolates (Figure 1). Using the interpretation criteria of PFGE patterns proposed by Tenover., et al. [19], the six strains of CRKP from different years were identified as the same strain.

Genomic characteristics of strains studied

The complete genome sequences of isolates 8297, 7213, 6106, 9168,9070 , and 7849 were successfully obtained by combining Illumina short-read and Nanopore long-read data. The results of the analysis indicated that all isolates carried blaKPC-2. Strain 8297 had one chromosome of 5,962,663 bp and seven plasmids. Strain 7213, 6106, 7849 and 9070 had six plasmids and chromosomes of 5,957;769 bp;

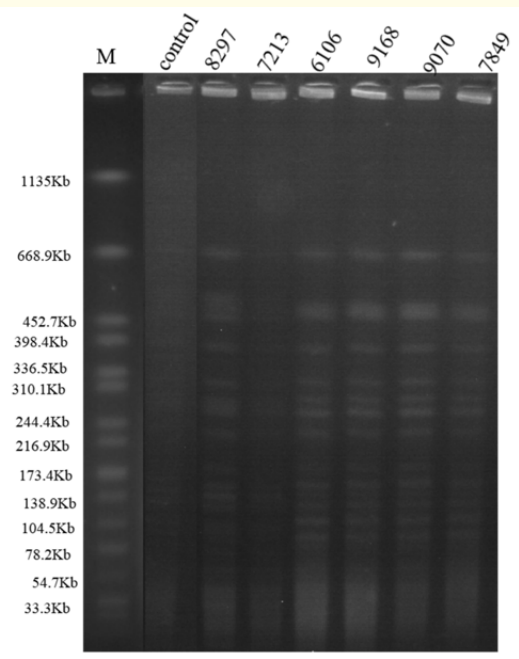

Figure1: Clustering of the $6 \mathrm{~K}$. pneumoniae isolates based on PFGE patterns.

5,961,238 bp; 5,987,968 bp and 5,986,653 bp, respectively. Strain 9168 had one chromosome of 5,624,028 bp and four plasmids. The plasmids which include blaKPC-2 are the same size in strain 6106, 7213 and 8297; all were isolated in 2017. The size of pKP9070_KPC and pKP7849_KPC are also the same, which were isolated in 2019.

Associated with the XDR phenotype, resistance determinants were identified in each strain (Table 1). The chromosomal and plasmidcarried determinants were relevant to the resistance of the tested antimicrobials, including $\beta$-lactams (blaKPC-2, blaCTX-M-65, blaSHV-12 and blaSHV-11), aminoglycosides (aadA2, aadA1 and $r m t B$ ), quinolones (qnrS1, oqxA and oqxB), chloramphenicol (catA2), doxycycline [tet(A)], trimethoprim-sulfamethoxazole (sul1, sul2, dfrA12 and dfrA14), fosfomycin $(f o s A)$ and macrolides $[m p h(A)]$. These genotypes were consistent with phenotypic test results (Supplement table 2).

\begin{tabular}{|c|c|c|c|c|c|c|c|c|c|c|c|}
\hline Year & $\begin{array}{c}\text { Patient } \\
\text { no. }\end{array}$ & Sex & $\begin{array}{c}\text { Age } \\
\text { (year) }\end{array}$ & $\begin{array}{c}\text { Admission } \\
\text { time }\end{array}$ & Cancer & $\begin{array}{c}\text { Neurosurgical } \\
\text { procedures }\end{array}$ & Source & $\begin{array}{c}\text { The } \\
\text { studied } \\
\text { strain } \\
\text { ID and } \\
\text { Source }\end{array}$ & $\begin{array}{l}\text { The date } \\
\text { of CRKPN } \\
\text { was first } \\
\text { detected }\end{array}$ & Treatment & Outcome \\
\hline \multirow{4}{*}{2017} & 1 & M & 48 & 12-Jul-17 & $\begin{array}{l}\text { Right frontal } \\
\text { glioma }\end{array}$ & Yes & $\begin{array}{l}\text { Ca, dn, } \\
\text { sf }\end{array}$ & 8297, Ca & 21-Jul-17 & CL, MI & $\begin{array}{c}\text { The } \\
\text { infection } \\
\text { is } \\
\text { controlled }\end{array}$ \\
\hline & 2 & $\mathrm{~F}$ & 70 & 11-Jul-17 & $\begin{array}{c}\text { Left } \\
\text { temporo-oc- } \\
\text { cipital triangle } \\
\text { glioma }\end{array}$ & Yes & sf, bl & $\begin{array}{c}\text { Not } \\
\text { studied }\end{array}$ & 21-Jul-17 & CL, MI, TGC & $\begin{array}{c}\text { The } \\
\text { infection } \\
\text { is } \\
\text { controlled }\end{array}$ \\
\hline & 3 & $\mathrm{~F}$ & 53 & 10-Jul-17 & $\begin{array}{c}\text { Giant } \\
\text { meningioma of } \\
\text { left sphenoid } \\
\text { crest }\end{array}$ & Yes & $\begin{array}{l}\text { sf, bl, } \\
\text { ps }\end{array}$ & $7213, \mathrm{sf}$ & 23-Jul-17 & CL, MI, TGC & $\begin{array}{c}\text { The } \\
\text { infection } \\
\text { is } \\
\text { controlled }\end{array}$ \\
\hline & 4 & $\mathrm{~F}$ & 70 & 20-Jun-17 & $\begin{array}{l}\text { Left cerebellar } \\
\text { tumor }\end{array}$ & Yes & $\mathrm{bl}, \mathrm{sf}$ & $6106, \mathrm{bl}$ & 6-Jul-17 & TGC & Died \\
\hline
\end{tabular}




\begin{tabular}{|c|c|c|c|c|c|c|c|c|c|c|c|}
\hline \multirow{3}{*}{2018} & 5 & M & 57 & 19-Jul-18 & $\begin{array}{c}\text { Left } \\
\text { cerebello- } \\
\text { pontine angle } \\
\text { tumor }\end{array}$ & Yes & $\mathrm{bl}, \mathrm{sf}$ & $9168, \mathrm{bl}$ & 3-Aug-18 & $\begin{array}{l}\text { TGC, MEM, } \\
\text { POY }\end{array}$ & Died \\
\hline & 6 & M & 70 & 10-Jul-18 & $\begin{array}{l}\text { Left } \\
\text { frontotempo- } \\
\text { ral glioma }\end{array}$ & Yes & $\begin{array}{l}\text { sp, bl, } \\
\text { sf }\end{array}$ & $\begin{array}{l}\text { Not stud- } \\
\text { ied }\end{array}$ & 20-Jul-18 & $\begin{array}{l}\text { C/S, LEV, } \\
\text { SXT }\end{array}$ & $\begin{array}{c}\text { The } \\
\text { infection } \\
\text { is } \\
\text { controlled }\end{array}$ \\
\hline & 7 & $\mathrm{~F}$ & 57 & 1-Aug-18 & Meningioma & Yes & $\begin{array}{c}\mathrm{dn}, \mathrm{bl}, \\
\text { sf }\end{array}$ & $\begin{array}{l}\text { Not stud- } \\
\text { ied }\end{array}$ & 10-Aug-18 & MI, TGC & $\begin{array}{c}\text { The } \\
\text { infection } \\
\text { is } \\
\text { controlled }\end{array}$ \\
\hline \multirow{3}{*}{2019} & 8 & M & 63 & 23-Jul-19 & Glioma & Yes & $\begin{array}{l}\mathrm{Ca}, \mathrm{bl}, \\
\text { sf }\end{array}$ & $9070, \mathrm{bl}$ & 31-Jul-19 & $\begin{array}{l}\text { MEM, TGC, } \\
\text { CL }\end{array}$ & Died \\
\hline & 9 & $\mathrm{~F}$ & 49 & 24-Jul-19 & $\begin{array}{l}\text { Epidermoid } \\
\text { galactoma }\end{array}$ & Yes & sf, bl & $7849, \mathrm{sf}$ & 16-Aug-19 & $\begin{array}{c}\text { TGC, CL, } \\
\text { POY }\end{array}$ & Died \\
\hline & 10 & M & 62 & 2-Aug-19 & $\begin{array}{l}\text { Right thalamic } \\
\text { tumor }\end{array}$ & Yes & $\begin{array}{l}\text { sp, bl, } \\
\text { sf }\end{array}$ & $\begin{array}{l}\text { Not stud- } \\
\text { ied }\end{array}$ & 20-Aug-19 & TGC, POY & $\begin{array}{c}\text { The } \\
\text { infection } \\
\text { is } \\
\text { controlled }\end{array}$ \\
\hline
\end{tabular}

Supplement Table 1: Characteristics of neurosurgical patients with nosocomial meningitis during the period 2017-2019.

Abbreviations: Ca: Catheter; dn: Drain; sf: cerebrospinal fluid; bl: blood; ps: pus; sp, sputum; cl: chloramphenicol; MI; minocycline; TGC: Tigecycline; MEM: Meropenem; POY, Polymyxin; C/S: cefoperazone/sulbactam; LEV: Levofloxacin; SXT: Trimethoprim/sulfamethoxazole.

\begin{tabular}{|c|c|c|c|c|c|c|c|c|c|c|c|c|c|c|c|c|c|}
\hline \multirow{2}{*}{ Year } & \multirow{2}{*}{ Patient } & \multicolumn{16}{|c|}{ MIC } \\
\hline & & GEN & AMP & FEP & FOX & CRO & TZP & SXT & LVX & CIP & MEM & IPM & AMK & CL & MI & POY & TGC \\
\hline \multirow{4}{*}{2017} & 1 & 16 & 32 & 32 & 32 & 64 & 128 & 8 & 8 & 4 & 16 & 16 & 64 & 8 & 4 & 0.25 & 1 \\
\hline & 2 & 16 & 32 & 32 & 32 & 64 & 128 & 8 & 8 & 4 & 16 & 16 & 64 & 8 & 4 & 0.25 & 0.5 \\
\hline & 3 & 16 & 32 & 32 & 32 & 64 & 128 & 8 & 8 & 4 & 16 & 16 & 64 & 4 & 4 & 0.25 & 0.25 \\
\hline & 4 & 16 & 32 & 32 & 32 & 64 & 128 & 4 & 8 & 4 & 16 & 16 & 64 & 8 & 4 & 0.25 & 0.25 \\
\hline \multirow{3}{*}{2018} & 5 & 16 & 32 & 32 & 32 & 64 & 128 & 0.5 & 8 & 4 & 16 & 4 & 64 & 32 & 4 & 0.125 & 0.25 \\
\hline & 6 & 16 & 32 & 32 & 32 & 64 & 128 & 0.5 & 8 & 4 & 16 & 16 & 64 & 32 & 4 & 0.5 & 1 \\
\hline & 7 & 16 & 32 & 32 & 32 & 64 & 128 & 8 & 8 & 4 & 16 & 16 & 64 & 32 & 4 & 0.5 & 1 \\
\hline \multirow{3}{*}{2019} & 8 & 16 & 32 & 32 & 32 & 64 & 128 & 4 & 16 & 4 & 16 & 16 & 64 & 8 & 16 & 0.25 & 0.5 \\
\hline & 9 & 16 & 32 & 32 & 32 & 64 & 128 & 4 & 16 & 4 & 16 & 16 & 64 & 8 & 16 & 0.125 & 0.5 \\
\hline & 10 & 16 & 32 & 32 & 32 & 64 & 128 & 4 & 16 & 4 & 16 & 16 & 8 & 32 & 16 & 0.125 & 0.25 \\
\hline
\end{tabular}

Supplement Table 2: Characteristics of antimicrobial susceptibilities in the ten patients.

Abbreviations: GEN: Gentamicin; AMP: ampicillin; FEP: Cefepime; FOX: Cefoxitin; CRO: Cefatriaxone; TZP: Piperacillin-Tazobactam; SXT: Trimethoprim-Sulfamethoxazole; LVX: Levofloxacin; CIP: Ciprofloxacin; MEM: Meropenem; IPM: Imipenem; AMK: Amikacin; CL: Chloramphenicol; MI: Minocycline; POY: Polymyxin; TGC: Tigecycline 


\begin{tabular}{|c|c|c|c|c|}
\hline Isolates & Source & Resistance determinants & $\begin{array}{l}\text { Chromosome } \\
\text { size }\end{array}$ & Plasmid size \\
\hline 8297 & ca & $\begin{array}{l}\text { aadA2 blaCTX-M- } 65 \text { blaKPC-2 blaSHV-12 } \\
\text { dfrA12 fosA mph(A) rmtB sul1 }\end{array}$ & $5962663 \mathrm{bp}$ & $\begin{array}{l}5485 \text { 939bp、229 339bp } 129531 \mathrm{bp} 、 \\
98 \text { 788bp(pKP8297_KPC) } 10060 \mathrm{bp} 、 5 \text { 596bp、 } 3 \\
410 \mathrm{bp}\end{array}$ \\
\hline 7213 & sf & $\begin{array}{l}\text { aadA2 blaCTX-M- } 65 \text { blaKPC-2 blaSHV-12 } \\
\text { dfrA12 fosA mph(A) rmtB sul1 }\end{array}$ & $5957769 b p$ & $\begin{array}{l}5485 \text { 163bp、228 622bp、129 540bp、 } \\
98 \text { 788bp(pKP7213_KPC) 、10 060bp、5 596bp }\end{array}$ \\
\hline 6106 & bl & $\begin{array}{l}\text { aadA2 blaCTX-M-65 blaKPC-2 blaSHV-12 } \\
\text { dfrA12 fosA mph(A) rmtB sul1 }\end{array}$ & 5961 238bp & $\begin{array}{c}5487 \text { 558bp 、229696bp 、 } 129540 \mathrm{bp} \text {. } \\
98 \text { 788bp(pKP6106_KPC)、 } 10 \text { 060bp 、 } 5 \text { 596bp }\end{array}$ \\
\hline 9168 & bl & $\begin{array}{l}\text { aadA1 blaCTX-M- } 65 \text { blaKPC-2 blaSHV-11 } \\
\text { blaSHV-12 catA2 fosA oqxA oqxB rmtB }\end{array}$ & $5624028 b p$ & $\begin{array}{c}5480 \text { 246bp、 } 128 \text { 126bp(pKP9168_KPC)、 } \\
10 \text { 060bp、5 596bp }\end{array}$ \\
\hline 9070 & bl & $\begin{array}{l}\text { QnrS1 aadA1 blaCTX-M-65 blaKPC-2 } \\
\text { blaSHV-11 } \\
\text { blaSHV-12 dfrA14 fosA rmtB sul2 tet(A) }\end{array}$ & $5986653 b p$ & $\begin{array}{c}5519453 \mathrm{bp} 、 217877 \mathrm{bp} 、 148968 \mathrm{bp}\left(\mathrm{pKP} 9070_{-}\right. \\
\text {KPC)、 } \\
82789 \mathrm{bp} 、 11970 \mathrm{bp} 、 5596 \mathrm{bp}\end{array}$ \\
\hline
\end{tabular}

Table 1: Molecular characteristics of the strains and their plasmids.

Abbreviations: Ca: Catheter; sf: Cerebrospinal Fluid; bl: Blood.

Plasmid and genome Phylogenetic analysis based WGS

According to the analysis, the plasmids harbouring blaKPC-2 (pKP6106_KPC, pKP7213_KPC and pKP8297_KPC) had the same sequence; pKP7849_KPC and pKP9070_KPC also had the same sequence. The detailed comparison of the strains is shown in Figure 2A. Comparing the plasmid sequences, the six isolates all had IncFII-type plasmids, which is proposed to promote the spread of the blaKPC gene in K. pneumoniae ST11 in China. Moreover, their blaKPC-2 genes had the same genetic environment in pKP6106_ KPC, pKP7213_KPC, pKP7849_KPC, pKP8297_KPC and pKP9070_ KPC; the exception was pKP9168_KPC, which differed (Figure 2B). pKP9168_KPC has the same identity as CP025463.1 as identified by nucleotide Blast; CP025463.1 is the accession number of strain p44-2, which was recovered from Huashan Hospital (Shanghai, China) in 2016. These analyses revealed the plasmids harbouring blaKPC-2 were the same between 2017 and 2019.
Phylogenetic analysis based on the k-mer algorithm shows that the strains isolated from 2017 (7213, 6106 and 8297) and 2019 (7849 and 9070) share certain phylogenetic relationships (Figure 3). 9168 was found to be closely related to the genomes of other strains.

\section{Discussion}

Bacterial meningitis in hospitals, especially intracranial infections caused by bacteria which resistant to carbapenems, is a lifethreatening complication in neurosurgery patients $[20,21]$. The most common pathogen of hospital-associated meningitis is Staphylococcus, and Gram-negative bacteria with multi-drug resistance and extensive drug resistance, including Acinetobacter baumannii, Pseudomonas aeruginosa, Escherichia coli and Klebsiella pneumoniae; CRKP is rarely isolated from CSF samples [12,13,22,23]. Nevertheless, in many countries, including Turkey, the United States and 


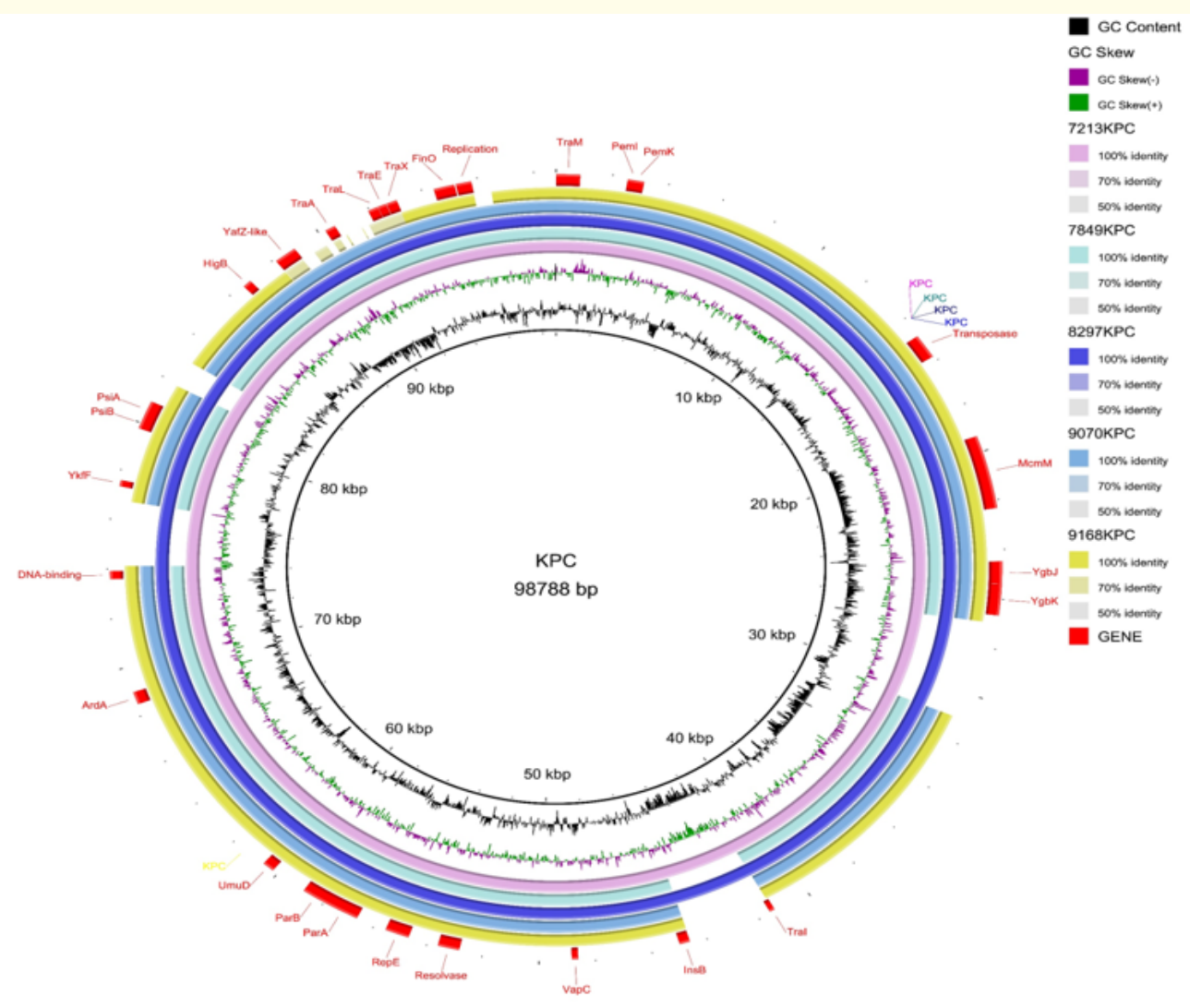

Figure 2A: Circular comparison of plasmids pKP6106-KPC, pKP7213_KPC, pKP8297_KPC, pKP7849_KPC, pKP9070_KPC and pKP9168_KPC, the interior loop is pKP6106_KPC.

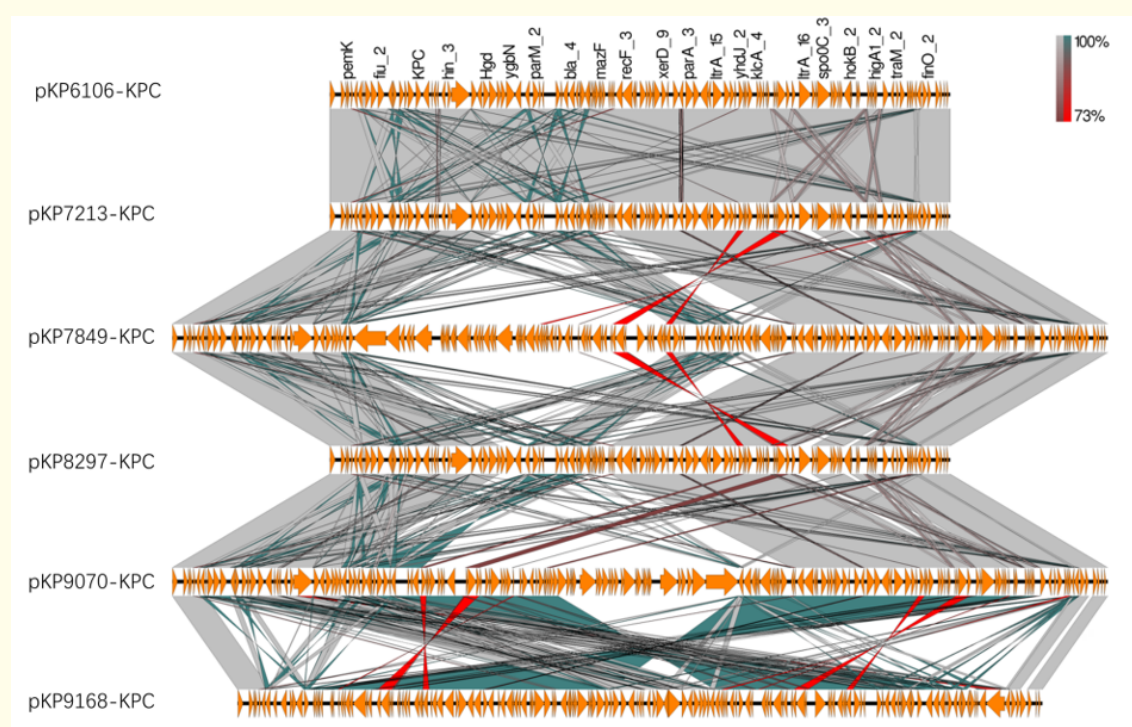

Figure 2B: Linear alignment of plasmids pKP6106-KPC, pKP7213_KPC, pKP8297_KPC, pKP7849_KPC, pKP9070_KPC and pKP9168_KPC, the arrows represent the direction of transcription.

Figure 2: Structure and analysis of KPC plasmids. 


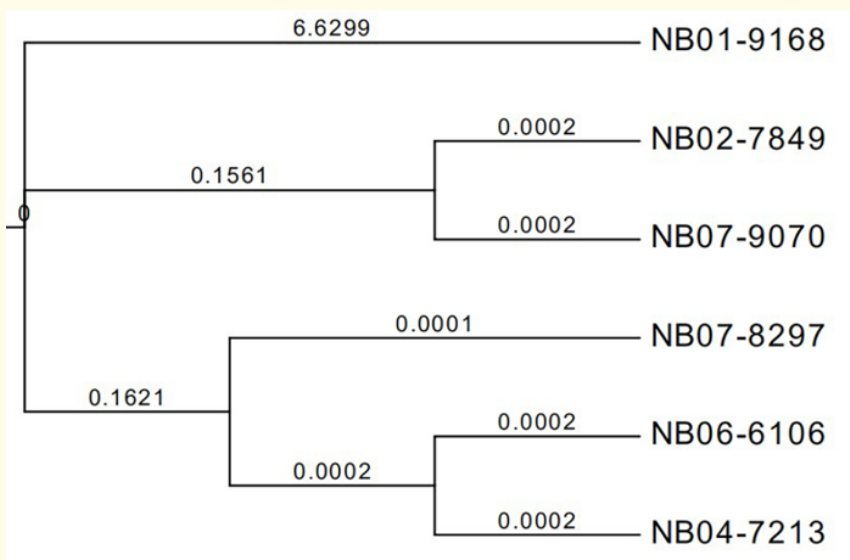

Figure 3: SNP analysis of strain 7213, 6106, 8297, 7849, 9070 and 9168, performed using kSNP. The maximum likelihood analysis of the core SNP matrix output for kSNP is performed in iTOL.

China, meningitis caused by CRKP neurosurgery has been reported [13,23-25]. To our knowledge, this is the first CRKP meningitis outbreak in cancer patients described in central China.

From July to September from 2017 to 2019, there were 3-4 cases of meningitis in a short period caused by CRKP in the neurosurgical ward of our hospital.Strictly implement infection control measures every year, including that all CRKP carriers should be isolated in a room under strict contact prevention measures and marked in the hospital information system; workers strengthen the disinfection of chlorhexidine in patient wards, And implement a culture of monitoring of hands and the environment; the hospital health nurse manager emphasizes the cleaning of high contact surfaces such as door handles, bedside lockers, chairs and bed rails to reduce cross transmission. Similarly, all exposed patients undergo rectal and pharynx screening once a week. However, despite these measures of surveillance, the samples from environmental and colonisation screening were negative for CRKP and the source of the infection could not be found.

It was very difficult to clarify transmission events between patients based on epidemiological data alone [26]. The combination of genetic information and clinical epidemiology can better explain the spread of outbreaks. Phylogenetic analysis based on the difference and SNP, in this study identified isolates exhibit closely relat- ed, and the difference in binding between the patient/department and SNP difference, the pathogen may be determined from a patient to another Patient transmission. The spread over three years suggests that CRKP may still be present in the hospital; the results of this study enforce the importance of continuing active surveillance cultures, and provide support for including healthcare workers, common environmental areas and jointly used medical devices in surveillance procedures. Increasing these protocols could help in limiting spread of CRKP and improving outbreak management.

Previous studies have shown, ST11 is a typical advantage of KPC-Kp clone, and has been widely reported in different parts of China [12,15,27-31]; all the strains identified in this study were ST11, and the plasmids carrying blaKPC were IncFII-type. Previous studies have demonstrated the diversity of plasmids carried by blaKPC, including IncFII, IncN, IncL/M, IncR and ColE1 groups, ranging in size from 10 to $300 \mathrm{~kb}$ [32-36]. IncF replicons may be further divided into FIA, FIB, FIC and FII groups. IncFII family plasmid present in a variety Enterobacter and plays an important role in the spread of antimicrobial resistance gene or the like as blaKPC $[28,37]$. There is a close correlation between ST11 KPC-Kp and IncFII-like plasmids, which indicates that ST11 KPC-Kp seems to be a good settler because it can capture IncFII-like plasmids that are widely spread in clinical settings [27]. It is known that horizontal gene transfer mediated by IncFII-like plasmids plays an important role in the pandemic spread of blaKPC-2 in Klebsiella pneumoniae ST11 in China. In addition, the genetic environment surrounding blaKPC-2 was investigated in the isolates collected in this study; the results suggest the genetic background of blaKPC-2 is relatively similar in all plasmids, as the blaKPC-2 genes were located within the same genetic context. Although the six isolates all carried IncFII-type plasmids, the size of the plasmid carrying the blaKPC-2 differed between isolates, indicating that the blaKPC-2 in the hospital strains may have been from various origins, spread throughout the hospital for many years. It is necessary to conduct a long-term retrospective genomic study of KPC-producing K. pneumoniae throughout the hospital to elucidate the evolution of KPCproducing K. pneumoniae.

Despite its significant results, this study did have limitations. Firstly, too few strains were studied; although the study contained strains isolated from different years, it is a retrospective analysis of medical records and therefore not all species and isolates from 
those periods were collected. The collected samples, whether from the environment, the patient, or the medical staff themselves did not have detected CRKP at the time; the source of CRKP has not been traced back to until now.

\section{Conclusions}

In summary, this study reports an outbreak in patients with nervous system tumours caused by closely related CRKP that lasted three years. When an outbreak occurs, the patient and the environment should be disinfected immediately, and the patient should be given appropriate treatment and be isolated; these measures can effectively contain the outbreak. In addition, the results of molecular analysis showed that ST11 and IncFII-type plasmids in CRKP have a close correlation and indicate that a long-term retrospective genomic study throughout the hospital for KPC-producing K. pneumoniae is necessary.

\section{Declarations}

Funding

This study was supported through Hospital Nursery Fund: Grant No. 2017011.

\section{Conflicts of Interest}

Competing interests (Not applicable).

\section{Ethics Approval}

This study obtained permissions from the Bioethics Committee of Affiliated Cancer Hospital of Zhengzhou University and Henan Cancer Hospital and participants (consent to participate was obtained from participants) to review patient records and use the data.

\section{Consent to Participate}

Yes.

\section{Consent to Publication}

Yes.

\section{Availability of Data and Material}

Yes.

\section{Code Availability}

Not applicable.

\section{Authors' Contributions}

MYS performed experimental work and drafted manuscript; WQX and YMC analyzed the study data; XKW and YYQ provided interpretation of data; QXX conceived the study and provided data interpretation. All authors read and approved the final manuscript. Bibliography

1. A Tsitsopoulos PP., et al. "Nosocomial bloodstream infections in neurosurgery: a 10-year analysis in a center with high antimicrobial drug-resistance prevalence". Acta Neurochirurgica 158.9 (2016): 1647-1654.

2. Kourbeti IS., et al. "Infections in patients with traumatic brain injury who undergo neurosurgery". British Journal of Neurosurgery 25.1 (2011): 9-15.

3. Orsi GB., et al. "Hospital-acquired infection surveillance in a neurosurgical intensive care unit". The Journal of Hospital Infection 64.1 (2006): 23-29.

4. Dziedzic T., et al. "Nosocomial infections and immunity: lesson from brain-injured patients". Critical Care 8.4 (2004): 266270 .

5. Gocmez C., et al. "Evaluation of risk factors affecting hospitalacquired infections in the neurosurgery intensive care unit". The International Journal of Neuroscience 124.7 (2014): 503508.

6. Laupland KB., et al. "Population-based assessment of intensive care unit-acquired bloodstream infections in adults: Incidence, risk factors, and associated mortality rate". Critical Care Medicine 30.11 (2002): 2462-2467.

7. Sablotzki A., et al. "Dysregulation of immune response following neurosurgical operations". Acta Anaesthesiologica Scandinavica 44.1 (2000): 82-87.

8. Tsitsopoulos PP., et al. "A 5-year epidemiological study of nosocomial bloodstream infections in a neurosurgery department". Infection Control and Hospital Epidemiology 31.4 (2010): 414417.

9. Hasbun R., et al. "Epidemiology of Meningitis and Encephalitis in the United States, 2011-2014”. Clinical Infectious Diseases: An Official Publication of the Infectious Diseases Society of America 65.3 (2017): 359-363.

10. Pereira PS., et al. "Update of the molecular epidemiology of KPC-2-producing Klebsiella pneumoniae in Brazil: spread of clonal complex 11 (ST11, ST437 and ST340)". The Journal of Antimicrobial Chemotherapy 68.2 (2013): 312-316. 
11. Tangden T and Giske CG. "Global dissemination of extensively drug-resistant carbapenemase-producing Enterobacteriaceae: clinical perspectives on detection, treatment and infection control". Journal of Internal Medicine 277.5 (2015): 501-512.

12. Almeida SM., et al. "Nosocomial meningitis caused by Klebsiella pneumoniae producing carbapenemase, with initial cerebrospinal fluid minimal inflammatory response". Arquivos de Neuro-Psiquiatria 72.5 (2014): 398-399.

13. Holyk A., et al. "Ceftazidime/avibactam use for carbapenemresistant Klebsiella pneumoniae meningitis: a case report". The Journal of Antimicrobial Chemotherapy 73.1 (2018): 254256.

14. Li R., et al. "Emergence of a novel conjugative hybrid virulence multidrug-resistant plasmid in extensively drug-resistant Klebsiella pneumoniae ST15". International Journal of Antimicrobial Agents 55.6 (2020): 105952.

15. Cheng L., et al. "Clonal dissemination of KPC-2 producing Klebsiella pneumoniae ST11 clone with high prevalence of oqxAB and rmtB in a tertiary hospital in China: results from a 3-year period". Annals of Clinical Microbiology and Antimicrobials 15 (2016): 1.

16. Wick RR., et al. "Unicycler: Resolving bacterial genome assemblies from short and long sequencing reads". PLoS Computational Biology 13.6 (2017): e1005595.

17. Gardner SN., et al. "kSNP3.0: SNP detection and phylogenetic analysis of genomes without genome alignment or reference genome”. Bioinformatics 31.17 (2015): 2877-2878.

18. Felsenstein J. "Evolutionary trees from DNA sequences: a maximum likelihood approach". Journal of Molecular Evolution 17.6 (1981): 368-376.

19. Tenover FC., et al. "Interpreting chromosomal DNA restriction patterns produced by pulsed-field gel electrophoresis: criteria for bacterial strain typing". Journal of Clinical Microbiology 33.9 (1995): 2233-2239.

20. Khan SA., et al. "Intrathecal and intraventricular antibiotics for postoperative Gram-negative meningitis and ventriculitis". Surgical Neurology International 8 (2017): 226.

21. Bardak-Ozcem S and Sipahi OR. "An updated approach to healthcare-associated meningitis". Expert Review of Anti-Infective Therapy 12.3 (2014): 333-342.

22. Yang KC., et al. "Occult community acquired Klebsiella pneumoniae purulent meningitis in an adult: A case report". Medicine 97.25 (2018): e11017.
23. Patrial YC., et al. "Post-neurosurgical meningitis caused by KPC-producing Klebsiella pneumoniae: report of two cases". Revista do Instituto de Medicina Tropical de Sao Paulo 61 (2019): e69.

24. Mermer S., et al. "Carbapenem-resistant Klebsiella pneumoniae meningitis: A case report". Journal of Chemotherphy 28.5 (2016): 454-455.

25. Chen Y and Liu L. "The treatment of nosocomial meningitis and brain abscess by carbapenem-resistant Klebsiella pneumonia”. British Journal of Neurosurgery (2019): 1-3.

26. Snitkin ES., et al. "Tracking a hospital outbreak of carbapenemresistant Klebsiella pneumoniae with whole-genome sequencing". Science Translational Medicine 4.148 (2012): 148ra116.

27. Fu P., et al. "Pandemic spread of blaKPC-2 among Klebsiella pneumoniae ST11 in China is associated with horizontal transfer mediated by IncFII-like plasmids". International Journal of Antimicrobial Agents 54.2 (2019): 117-124.

28. Chen L., et al. "Carbapenemase-producing Klebsiella pneumoniae: molecular and genetic decoding". Trends in Microbiology 22.12 (2014): 686-696.

29. Chen L., et al. "Complete sequence of a bla (KPC-2)-harboring IncFII (K1) plasmid from a Klebsiella pneumoniae sequence type 258 strain". Antimicrobial Agents and Chemotherapy 57.3 (2013): 1542-1545.

30. Shen P., et al. "Molecular dissection of blaKPC-2-bearing plasmids evolving in Klebsiella pneumoniae isolated at one teaching hospital in Shanghai, China". FEMS Microbiology Letters 363.15 (2016): 1-5.

31. Wang LH., et al. "Diversity of the Genetic Environment of the blaKPC-2 Gene Among Klebsiella pneumoniae Clinical Isolates in a Chinese Hospital". Microbial Drug Resistance 22.1 (2016): 15-21.

32. Gootz TD., et al. "Genetic organization of transposase regions surrounding blaKPC carbapenemase genes on plasmids from Klebsiella strains isolated in a New York City hospital". Antimicrobial Agents and Chemotherapy 53.5 (2009): 1998-2004.

33. Andrade LN., et al. "Dissemination of blaKPC-2 by the spread of Klebsiella pneumoniae clonal complex 258 clones (ST258, ST11, ST437) and plasmids (IncFII, IncN, IncL/M) among Enterobacteriaceae species in Brazil". Antimicrobial Agents and Chemotherapy 55.7 (2011): 3579-3583. 
34. Kassis-Chikhani N., et al. "Complete nucleotide sequence of the first KPC-2- and SHV-12-encoding IncX plasmid, pKpS90, from Klebsiella pneumoniae". Antimicrobial Agents and Chemotherapy 57.1 (2013): 618-620.

35. Cuzon G., et al. "Worldwide diversity of Klebsiella pneumoniae that produce beta-lactamase blaKPC-2 gene". Emerging Infectious Diseases 16.9 (2010): 1349-1356.

36. Chi X., et al. "Genomic Analysis of A KPC-2-Producing Klebsiella Pneumoniae ST11 Outbreak from A Teaching Hospital In Shandong Province, China". Infection and Drug Resistance 12 (2019): 2961-2969.

37. Villa L., et al. "Replicon sequence typing of IncF plasmids carrying virulence and resistance determinants". The Journal of Antimicrobial Chemotherapy 65.12 (2010): 2518-2529.

\section{Assets from publication with us}

- Prompt Acknowledgement after receiving the article

- Thorough Double blinded peer review

- Rapid Publication

- Issue of Publication Certificate

- High visibility of your Published work

Website: www.actascientific.com/

Submit Article: www.actascientific.com/submission.php

Email us: editor@actascientific.com

Contact us: +919182824667 